\title{
Inactivation of Bacillus Subtilis Spores by a Combination of Hydrostatic High-Pressure and Pulsed Electric Field Treatments
}

\author{
Akihiko SASAGAWA ${ }^{1}$ \\ Jun HOSHINO
}

\author{
Akira YAMAZAKI ${ }^{2, *}$
}

Takayuki OHSHIMA ${ }^{3}$
Atsushi KOBAYASHI ${ }^{2}$

Masayuki SATO ${ }^{3}$

\author{
Tomoyuki FUJII
}

Akifumi YAMADA $^{1}$

\begin{abstract}
The effect of the combination of hydrostatic high-pressure (HHP) and pulsed electric field (PEF) treatments on the inactivation of Bacillus subtilis spores was investigated, using suspensions prepared by suspending the spores in various solutions at about $10^{8}$ spores/ml. When the PEF and HHP treatments were successively conducted in that order, the results were compared with the case where either was conducted alone. The combined processing achieved a $7.1 \mathrm{log}$ reduction in the viable spore counts at maximum. Low-temperature storage after the combined processing further led to reduced germination, and finally completes inactivation in 3 days. It was confirmed by phase-contrast microscopy that the non-treated spores gradually turned into phase-dark spores and finally germinated and changed into vegetative cells, while the spores subjected to PEF/HHP treatment did not transfer to the phase-dark stage, meaning no germination.

[hydrostatic high-pressure (HHP), pulsed electric field (PEF), combined effect, Bacillus subtilis spore, sterilization, germination]
\end{abstract}

\section{Introduction}

Food products are usually sterilized by heat treatment. In particular, to sterilize heat-resistant bacterial spores such as the Bacillus and Clostridium species, the sterilization temperature is required to be as high as $100^{\circ} \mathrm{C}$ or more. However, heat treatment has undesirable side effects, e.g., changes in flavor, taste and texture and alteration of ingredients.

To eliminate these side effects of heat treatment, new non-thermal technologies such as hydrostatic high- pressure (HHP) treatment[1-3], pulsed electric field (PEF) treatment[4, 5], supercritical $\mathrm{CO}_{2}$ treatment[6], and a combination of these treatments have become a focus of food scientists' attention because these technologies have the potential to sterilize or inactivate the heat-resistant spores without using heat energy [7, 8].

HHP technology can inactivate most vegetative cells in 10 to 30 minutes by pressure application of $600 \mathrm{MPa}$ [7]. However, Nakayama et al.[9] found that Bacillus subtilis was not inactivated even by pressure application of $981 \mathrm{MPa}$ for 40 minutes. Wuytack and

\footnotetext{
${ }^{1}$ Nagaoka University of Technology, 1603-1 Kamitomioka-machi, Nagaoka-shi, Niigata 940-2188

${ }^{2}$ Research Institute, Echigoseika Co., Ltd., 1003-1 Takanashi-machi, Ojiya-shi, Niigata 947-0193

${ }^{3}$ Department of Biological and Chemical Engineering, Gunma University, 1-5-1 Tenjin-cho, Kiryu-shi, Gunma 376-8515

${ }^{4}$ Department of Food Science, Niigata University of Pharmacy and Applied Life Sciences, 265-1 Higashishima Niigata-shi, Niigata 956-8603

*Electronic address: kenkyu@echigoseika.co.jp
} 
Michiels reported that the inactivation of $B$. subtilis spores was maximally $80 \%$ when the spore suspensions adjusted to $\mathrm{pH} 3-8$ were subjected to HHP treatment of $600 \mathrm{MPa}$ for 20 minutes at $40^{\circ} \mathrm{C}[2]$.

The PEF technique is typically used to import DNA into mammalian cells, and applications to plant protoplasts and yeast have been reported[10]. Yonemoto et al.[4] reported that the spores of B. subtilis were highly resistant to PEF showing little decrease in viability, although the viability of vegetative cells was sharply lowered.

In light of the above reports, HHP or PEF treatment has a sterilization effect on vegetative cells, but the complete inactivation of spores is considered difficult when non-thermal HHP or PEF treatment is conducted alone.

In this study, therefore, various suspensions of $B$. subtilis spores were subjected to a combination of HHP and PEF treatments, and the influences of the respective treatments on the inactivation of the spores were investigated. In addition, the suspensions were stored at low temperature for a long period after the completion of the combined processing. During the storage period, the number of viable spores was counted, and the state of spores was observed using a phase-contrast microscope to discuss the factors contributing to the inactivation.

\section{Materials and Methods}

\subsection{Spore culturing}

B. subtilis NBRC3007 was chosen for the experiments and obtained from the National Institute of Technology and Evaluation.

B. subtilis strain was pre-cultivated overnight in nutrient broth (Oxoid, Basingstoke, UK) at $35^{\circ} \mathrm{C}$. These were subsequently inoculated in the culture medium newly prepared and grown at $35^{\circ} \mathrm{C}$ for 7 days. The harvested culture was heated to $80^{\circ} \mathrm{C}$ for 30 minutes to sterilize only the vegetative cells, and was rapidly cooled. The culture was then centrifuged at 4,000 rpm for 20 minutes at $20{ }^{\circ} \mathrm{C}$ using a centrifugal separator (H-100B, Kokusan Co., Ltd.). The precipitated spore pellets were washed with $1 / 15 \mathrm{M}$ phosphate buffer solution ( $\mathrm{pH}$ 7.0) and centrifuged again. This washing procedure was repeated three times. The concentration of the obtained spore suspension was adjusted to
$10^{9}$ spores $/ \mathrm{ml}$ using a phosphate buffer solution. After the spore suspension was finally heated to $80^{\circ} \mathrm{C}$ for 30 minutes, the solution was tightly sealed in a non-rigid plastic bag and preserved by freezing at $-80^{\circ} \mathrm{C}$ until use.

\subsection{Sample preparation}

The frozen spore suspension in the bag was thawed in cold water before use. For the experiments, the spore suspensions were prepared by adding the spore solution separately to $0.1 \%$ aqueous $\mathrm{NaCl}$ solution (hereinafter referred to as $\mathrm{NaCl}$ solution), 1/15 $\mathrm{M}$ phosphate buffer solution of $\mathrm{pH} 7.0$ (hereinafter referred to as buffer solution ( $\mathrm{pH} 7.0)$ ), $0.5 \mathrm{M}$ acetate buffer solution of $\mathrm{pH} 5.0$ (hereinafter referred to as buffer solution ( $\mathrm{pH}$ 5.0)) 0.5 $\mathrm{M}$ acetate buffer solution of $\mathrm{pH} 3.3$ (hereinafter referred to as buffer solution ( $\mathrm{pH} 3.3)$ ), and a commercially available orange juice of $\mathrm{pH} 3.9$, to obtain the respective suspensions with a concentration of $10^{7}-10^{8}$ spores $/ \mathrm{ml}$.

\subsection{PEF treatment}

The system for the PEF treatment in the experiments is schematically shown in Fig. 1[11]. This system was originally designed by making slight improvements to a conventional system as reported by Ohshima et al.[11, 12]. As shown in Fig. 2, the PEF treatment chamber[13] was composed of two acrylic resin pipes with a length of $55 \mathrm{~mm}$ : an inner pipe of $19 \mathrm{~mm}$ outside diameter, was inserted into an outer pipe with a gap of

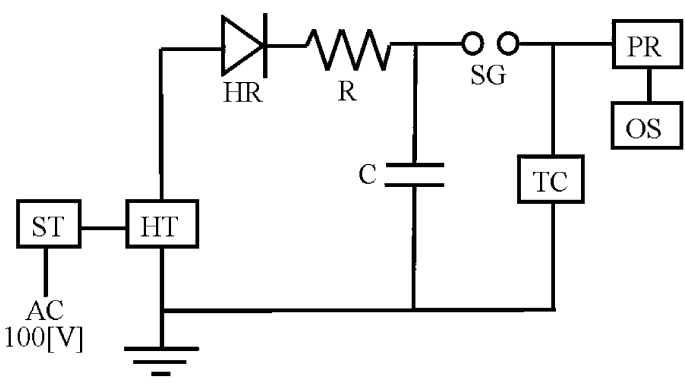

ST: slide transformer HR: high voltage rectifier $\mathrm{C}$ : capacitor TC: treatment chamber OS: digital oscilloscope
HT: high voltage transformer $\mathrm{R}$ : high voltage resistor SG: spark gap PR: high voltage probe
Fig. 1. Schematic diagram of PEF system. 
$3.5 \mathrm{~mm}$. Two platinum electrode wires were wound around the inner pipe in a spiral, with the adjacent electrode wires being disposed at intervals of about $8 \mathrm{~mm}$. One of the wires was connected to a pulsed power supply, and the other was connected to the ground. The PEF treatment was conducted under the following conditions: pulsed voltage, $12 \mathrm{kV}$; frequency, $50 \mathrm{~Hz}$; capacitor, capacitance of $8 \mathrm{nF}$. The voltage and current waveforms were observed using a digital oscilloscope (DL1620 701605-AC-M-J1/B5, Yokogawa Electric Corporation) with a high-voltage probe (P6015A, Tektronix, Inc.) when voltage was applied across the electrodes with the chamber filled with a sample.

A flask containing $200 \mathrm{ml}$ of the sample was maintained at $55^{\circ} \mathrm{C}$ in a water bath. The flask and the PEF treatment chamber were connected by Teflon tubing. The sample was circulated using a tube pump at a flow rate of $160 \mathrm{ml} / \mathrm{min}$. After the PEF treatment, the sample was removed from the flask in an aseptic manner and the viable spore counts were determined.

Marsili et al.[14] reported that significant reductions in microbial population were achieved, demonstrating the effectiveness of using the plasma discharge process to treat contaminated liquids. Plasma discharge was not generated in this PEF treatment chamber.

\subsection{HHP treatment}

After the spore sample $(200 \mathrm{ml})$ was packed into a non-rigid aseptic plastic bag, the air in the bag was minimized by deaeration, and the bag was then heat-sealed. The sample thus obtained was placed in a high-pressure

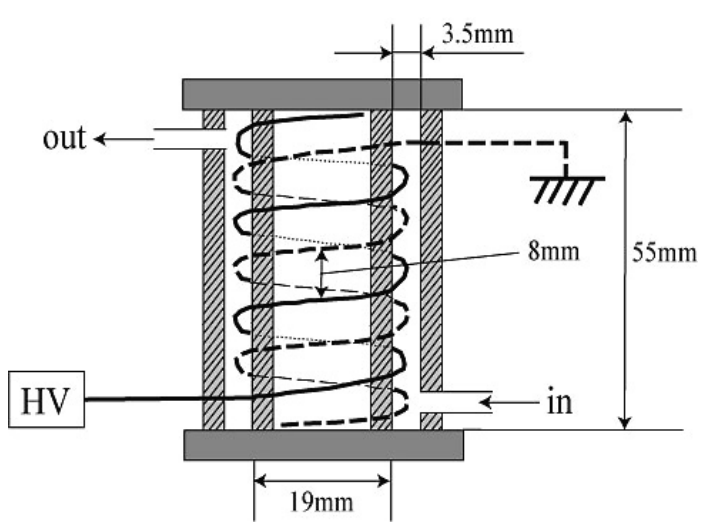

Fig. 2. Schematic diagram of PEF treatment chamber. cylindrical chamber with a diameter of $0.11 \mathrm{~m}$ and a depth of $0.40 \mathrm{~m}$ of the hydrostatic pressing system (Ishikawajima-Harima Heavy Industries Co., Ltd.). This system was specially designed for food products to control the temperature inside the chamber by circulating warm water around the outside[1]. The HHP treatment was conducted at $700 \mathrm{MPa}$ and $55^{\circ} \mathrm{C}$ for 10 minutes, with the applied pressure increased or decreased at a rate of $200 \mathrm{MPa} / \mathrm{min}$. The increase in temperature caused by adiabatic compression was about $2.5^{\circ} \mathrm{C} / 100 \mathrm{MPa}$ during the HHP treatment. The temperature of the HHP treatment was recorded using a thermocouple attached to the bottom of the chamber.

\subsection{Combination of HHP and PEF treatments}

The HHP and the PEF treatments were successively conducted so that the subsequent treatment was readily initiated within about 10 minutes after the previous treatment in order to confirm the combined effect of the PEF and HHP treatments on the inactivation of B. Subtilis spores. Hereafter, the process where PEF treatment is followed by HHP treatment will be referred to as PEF/HHP treatment; while the process where HHP treatment is followed by PEF treatment, as HHP/PEF treatment.

\subsection{Low-temperature storage of treated samples after treatment}

The influence of the HHP and/or PEF treatment on the subsequent germination of spores was examined by keeping the treated and non-treated samples in a refrigerator at $5^{\circ} \mathrm{C}$ over two weeks, and determining the viable spore counts after a lapse of a given number of days.

\subsection{Measurement of viable cell counts}

Ten-fold serial dilutions were prepared using physiological saline solution. The dilutions were inoculated onto a plate count agar (PCA). The plates were incubated at $35^{\circ} \mathrm{C}$ for 48 hours prior to counting. Colonyforming units on the culture plate were counted as viable cell counts and expressed in a logarithm. All experiments were performed in triplicate or more, and the mean was obtained from the results with the standard deviation taken into consideration. 


\subsection{Microscopic observation}

The state of the spores contained in the treated samples and non-treated samples was observed by a phasecontrast fluorescence microscope equipped with a digital camera (PM-10M, Olympus Corporation) [2, 15]. Specifically, a portion $(4 \mu \mathrm{L})$ of each sample was dropped on a Petri dish previously coated with the PCA. A lid was put on the dish to prevent moisture evaporation, and the culture was conducted at $35^{\circ} \mathrm{C}$. After a lapse of a given period, the dish was removed from the thermostat to observe the spores under phase-contrast microscopy.

\section{Results and Discussion}

\subsection{Pulse waveforms in PEF treatment}

The voltage and current waveforms applied across the electrodes via the sample suspension are shown in Fig. 3.

When voltage is placed between the electrodes in an RC series circuit (Fig. 1) where the sample suspension has electrical resistance $(R)$, voltage $(V)$ between the electrodes (Fig. 2) is expressed by the following
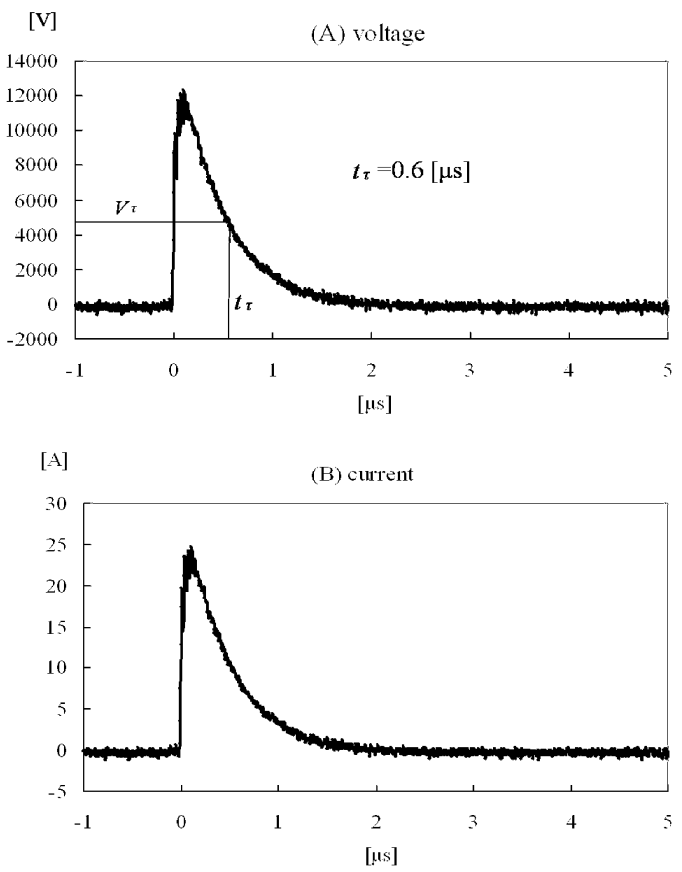

Fig. 3. Waveforms of voltage (A) and current (B) in PEF treatment. equation[4]:

$$
V=V_{0} \cdot \exp (-t / \tau)
$$

where $\tau$ is the time constant $(\tau=R C)$ of an electric pulse applied to the sample suspension.

In this experiment, voltage $\left(V_{\tau}\right)$ obtained when $t=$ $\tau$ is $V_{0} \cdot e^{-1}=4415$ [V]. Namely, time $\left(t_{\tau}\right)$, a representative value of the pulse waveform, can be read as approximately $0.6 \mu$ s from Fig. 3 . Since the frequency was set at $50 \mathrm{~Hz}$, the pulsed voltage was applied to the suspension at intervals of $20 \mathrm{~ms}$.

Applied energy $(U)$ is defined as energy per unit volume of the sample by the following equation[13, 16]:

$$
U=(1 / 2) \cdot C \cdot V^{2} \cdot F \cdot T \cdot v^{-1}[\mathrm{~J} / \mathrm{ml}],
$$

where $C$ is the capacitance of capacitor $[\mathrm{F}], V$ is pulsed voltage $[\mathrm{V}], F$ is frequency $[\mathrm{Hz}], T$ is treatment time [s], and $v$ is volume to be treated $[\mathrm{ml}]$.

Consequently, the applied energy in this experiment was calculated to be $173[\mathrm{~J} / \mathrm{ml}]$.

\subsection{Change in viable spore counts immediately after each treatment}

Fig. 4 shows the change in viable spore count in each sample. The viable spore count of each suspension was determined immediately after the PEF, HHP, $\mathrm{PEF} / \mathrm{HHP}$ or HHP/PEF treatment. The viable spore count immediately after each treatment is a value corresponding to the storage time of zero.

\subsubsection{Change in viable spore counts by PEF treat- ment}

Immediately after the PEF treatment, the sample suspension using $\mathrm{NaCl}$ solution showed a $0.3 \mathrm{log}$ reduction in viable spore count compared with the non- treated sample. There was no substantial decrease in the viable spore counts in any buffer solutions regardless of the $\mathrm{pH}$ value. No reduction in the viable spore count was observed in the orange juice.

Heinz et al.[17] reported that the energy of 155 $\mathrm{kJ} / \mathrm{kg}$ was necessary to inactivate $B$. subtilis vegetative cells by one order of magnitude. Yonemoto et al.[4] reported that the spores still exhibited high resistance to the applied energy of $146 \mathrm{~J} / \mathrm{ml}$ although the activity of the vegetative cells was reduced by the same applied energy. The applied energy was $173 \mathrm{~J} / \mathrm{ml}$ in our experiments. Considering that the applied energy was almost 
the same as that in Yonemoto et al.[4], our results that the PEF a treatment did not significantly inactivate the spores are convincing. Ho et al.[18] proposed a treatment using instant-charge-reversal pulses. Based on the design of Ho et al., Marquez et al.[5] reported a 3.4 log reduction in spore count by high-voltage pulsed electric field (HVPEF) treatment. Even if the above-mentioned reports are taken into consideration, it is found to be difficult to completely inactivate $B$. subtilis spores only by PEF treatment.

\subsubsection{Change in viable spore counts by HHP treat-} ment

The viable spore counts were reduced in all the suspension samples immediately after the HHP treatment. The viable spore counts in the buffer solution ( $\mathrm{pH} 7.0)$ were lower than those in the $\mathrm{NaCl}$ solution. The lower the $\mathrm{pH}$ of the buffer solution, the lower the viable spore counts obtained. It is therefore considered that the lower the $\mathrm{pH}$ of the solution in which the spores are suspended, the greater the inactivation effect on $B$. subtilis spores achieved by the HHP treatment. In the case of the orange juice, the spore count showed about a $5.6 \log$ reduction. The maximum reduction, i.e., about $6.4 \mathrm{log}$ reduction, was observed in the buffer solution of $\mathrm{pH} 3.3$, the lowest $\mathrm{pH}$ value.
Taki et al.[3] found that the HHP treatment of $600 \mathrm{MPa}$ at $45^{\circ} \mathrm{C}$ and $60^{\circ} \mathrm{C}$ achieved 5 and $6 \log$ reductions in the viable spore counts, respectively. Furthermore, there have been various reports on the combined effects of high-pressure and heat treatments on bacterial spores[1, 7, 19]. Judging from the abovementioned reports, however, complete inactivation of the spores is considered difficult only by non-thermal HHP treatment.

\subsubsection{Change in viable spore counts by PEF/HHP treatment}

The combined processing where the HHP treatment followed the PEF treatment achieved a 1.8 log reduction in the viable spore counts in the $\mathrm{NaCl}$ solution, a $7.1 \mathrm{log}$ reduction in the buffer solution $(\mathrm{pH} 3.3)$, which is the maximum reduction among these samples, and about a $6.5 \mathrm{log}$ reduction in the orange juice $(\mathrm{pH} 3.9)$. The $\mathrm{PEF} / \mathrm{HHP}$ treatment was found to be more effective in the inactivation of the spores than the PEF or HHP treatment.

Sara et al.[8] investigated the synergistic effect of $\mathrm{PEF}$ treatment and supercritical $\mathrm{CO}_{2}$ treatment $\left(\mathrm{SC}-\mathrm{CO}_{2}\right)$ on the inactivation of $B$. cereus spores, and concluded that a $0.5 \log$ reduction in the viable spore counts was obtained by PEF treatment alone, while a further $3 \mathrm{log}$

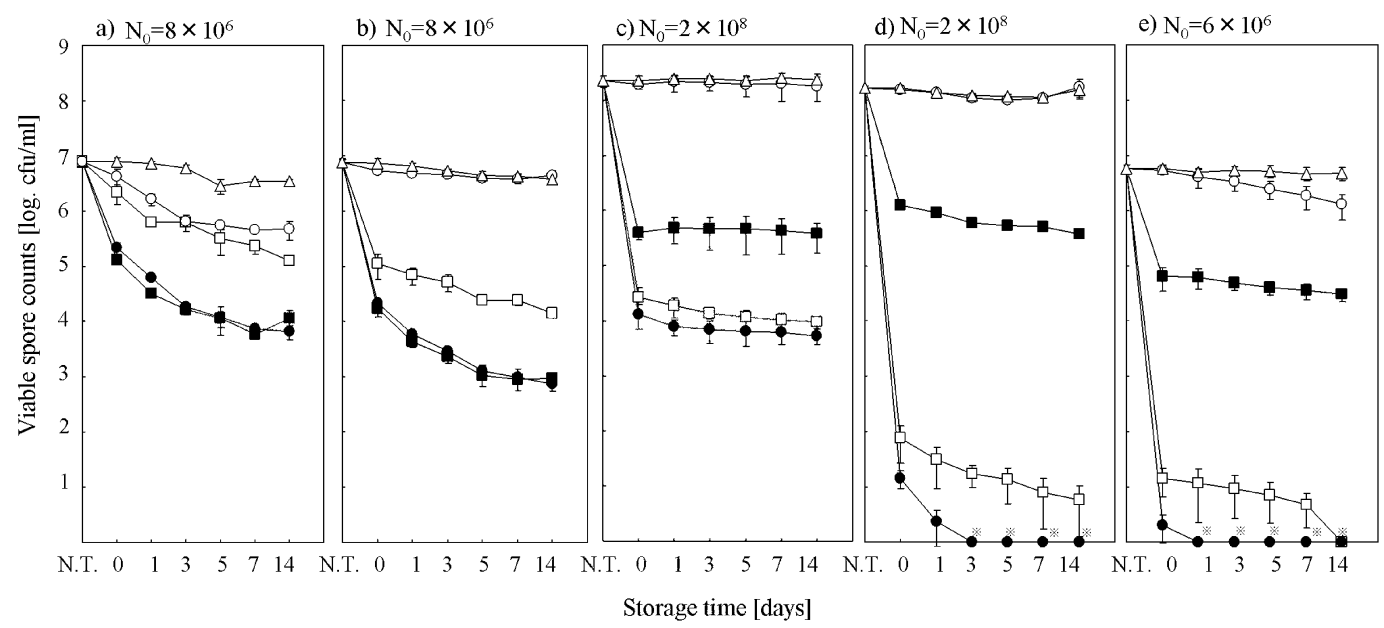

Fig. 4. Effect of PEF and HHP treatment on viable spore counts of B. subtilis with storage time at $5^{\circ} \mathrm{C}$. a), $\mathrm{NaCl}$ solution; b), Buffer solution ( $\mathrm{pH} 7.0)$; c), Buffer solution ( $\mathrm{pH}$ 5.0); d), Buffer solution ( $\mathrm{pH} 3.3$ ); e), Orange juice (pH 3.9). N.T., Nontreatment.

$\triangle$, Control; $\bigcirc$, PEF $\left(12 \mathrm{kV}\right.$ at $55^{\circ} \mathrm{C}$ for $\left.20 \mathrm{~min}\right) ; \square, \mathrm{HHP}\left(700 \mathrm{MPa}\right.$ at $55^{\circ} \mathrm{C}$ for $\left.10 \mathrm{~min}\right) ; \mathbf{P}, \mathrm{PEF} / \mathrm{HHP} ; \mathbf{\square}, \mathrm{HHP} / \mathrm{PEF}$. $※$, Non-detected. 
reduction was achieved when the PEF treatment was followed by $\mathrm{SC}-\mathrm{CO}_{2}$ treatment. It was also confirmed by SEM that the spores after PEF treatment had cracks or folds on their surfaces and their shape appeared less spherical compared with the control B. cereus spores, and the spores were further damaged by the subsequent $\mathrm{SC}-\mathrm{CO}_{2}$ treatment. They indicated that the morphological changes of the spores were closely related to the 3 log reduction in spore counts. Yonemoto et al.[4] also confirmed by electron microscopic observation that $B$. subtilis spores treated by PEF had cracks on their surfaces and black granules were crushed in an increased number of spores.

The energy applied to the spores in our experiments was almost the same as that in Yonemoto et al. It is therefore considered that the spores had cracks on their surfaces due to the PEF treatment, which accelerated the inactivation of the spores when the HHP treatment was subsequently conducted.

\subsubsection{Change in viable spore counts by HHP/PEF treatment}

To investigate the influence of the treatment sequence on spore viability, $\mathrm{HHP} / \mathrm{PEF}$ and $\mathrm{PEF} / \mathrm{HHP}$ treatments were conducted. In the $\mathrm{NaCl}$ solution and neutral buffer solution, the presence of some synergistic effect was evident from the fact that the viable spore counts were still lower compared with where either HHP or PEF treatment was conducted alone. However, in sharp contrast to the PEF/HHP treatment, the viable spore counts after the HHP treatment increased due to the subsequent PEF treatment in the low-pH-range suspensions, as shown in Fig. 4c)-4e). In other words, the graphs show that some of the spores were inactivated by the HHP treatment and their activity was thereafter recovered by the PEF treatment. Among these three kinds of solution in the low $\mathrm{pH}$ range, although the spores tended to be more efficiently inactivated by the HHP treatment as the $\mathrm{pH}$ of the solution became lower, the activity of the spores recovered after the subsequent PEF treatment.

Wuytack and Michiels[2] conducted HHP treatments at $\mathrm{pH} 3-8$ and suggested that the HHP treatment promoted the replacement of ions $\left(\mathrm{Ca}^{2+}, \mathrm{Na}^{+}, \mathrm{Mg}^{2+}\right.$, $\mathrm{Mn}^{2+}$, etc.) contained in the spores with hydrogen ions to form "H-spores" in the low $\mathrm{pH}$ range $(<5.0)$, thereby leading to enhanced heat inactivation of the spores. The results of this experiment indicate that the activation of spores by PEF treatment after HHP treatment may be associated with the formation of $\mathrm{H}$-spores.

Grahl and Markl[20] reported that some enzymes were readily inactivated and others were not when raw milk was subjected to PEF treatment. Bendicho et al.[21] reported that the protease activity derived from $B$. subtilis varied depending on the type of solution in which the enzyme is contained in PEF treatment. Furthermore, it is reported by Ho et al.[22] that when eight different kinds of enzyme solution were subjected to PEF treatment, the activities of lysozyme and pepsin increased.

With the above-mentioned reports taken into consideration, enzymes can be activated by PEF treatment. In this study, some spores once inactivated by the HHP treatment were germinated by the subsequent PEF treatment, suggesting the enzymes responsible for the germination could be activated by the PEF treatment.

\subsection{Change in viable spore counts during low- temperature storage after each treatment}

Each sample was stored at $5^{\circ} \mathrm{C}$ for 2 weeks immediately after each treatment, and changes in viable spore counts during the storage period were observed as shown in Fig. 4.

During the storage period, a $1.5 \log$ reduction in viable spore counts was observed in the $\mathrm{NaCl}$ solution and the buffer solution ( $\mathrm{pH}$ 7.0) after the combined processing of PEF and HHP. On the other hand, a substantial reduction was not observed while the buffer solution ( $\mathrm{pH}$ 5.0), buffer solution ( $\mathrm{pH}$ 3.3) and orange juice ( $\mathrm{pH}$ 3.9) were stored after HHP/PEF treatment. In the case of the buffer solution $(\mathrm{pH} 3.3)$ and orange juice ( $\mathrm{pH}$ 3.9) treated by PEF/HHP (Fig. 4d) and 4e)), the number of viable spores decreased to non-detectable levels in 1 to 3 days, indicating complete inactivation.

Hayakawa et al.[23, 24] reported that sterilization of B. stearothermophilus spores by high-pressure treatment was assumed to be determined by water permeability through the spore coat. Wallen et al.[25] referred to the storage conditions for the spores from the viewpoint of chemical resistance, and pointed out that the life expectancy of aging spores was influenced both by 
internal, hereditary considerations and by various external environmental factors[25].

In this study, the spores were completely inactivated by the PEF/HHP treatment during the extended period of storage time, and the mechanisms could be explained as follows:

(1) The spore coat is physically broken to form passages for liquid by the PEF treatment, so that the passages allow liquid to penetrate the inside of the spores at the subsequent HHP treatment.

(2) In the application of HHP treatment, the liquid surrounding the spores plays the role of a pressurizing medium when penetrating the spore cells, and the low-pH liquid promotes the inactivation of enzymes when mixing with the intracellular fluid.

(3) The storage time required to achieve complete inactivation is regarded as a delay time during which the low-pH liquid acts on the particular enzymes responsible for the process of germination after penetrating the intracellular fluid in the spores.

Namely, it is suggested that the storage time required for reducing the viable spore counts could be a delay time during which the acidic solution penetrating the inside of the spores inactivates the enzymes responsible for germination.

\subsection{Results of Phase-contrast microscopic obser- vation}

The spores suspended in the acidic buffer was dropped onto the PCA immediately after the PEF/HHP treatment and incubated at $35^{\circ} \mathrm{C}$. In the course of incuba- tion, the germination of the spores was checked by phase-contrast microscopic observation as shown in Fig. 5.

The non-treated spores (Fig. 5a)) turned into phase-dark spores in one hour (Fig. 5b)), germinated in two hours (Fig. 5c)), and grew finally into vegetative cells in the form of elongated rods in three hours (Fig. 5d)). In contrast to this, the spores subjected to PEF/HHP treatment did not turn into phase-dark spores even after 24 hours (Fig. 5h)).

Based on phase-contrast microscopic observation, Leuschner and Lillford[26] reported that the spores appeared to have a gloss due to light refraction before germination, while the phase brightness was lost by the hydration of the structural compartments in the spores at germination. The same authors discussed that the process of germination involved two phases according to the stage of germination and that the length of time when the phase-bright spores turned into phase-dark spores was dependent on the environment, especially the temperature[15]. Furthermore, germination involves the activities of lytic transglycosylase, glucosamidase and the like[27]. Leuschner and Lillford[15] revealed that the length of time required to complete the phase change varied depending on the incubation temperature, suggesting the involvement of enzymatic activity at the phase change in the process of germination.

In our experiments, the pressure used for the HHP treatment was $700 \mathrm{MPa}$, which is capable of inactivating typical enzymes. In addition, the spores were inactivated by PEF/HHP treatment. Therefore, it was indicated that

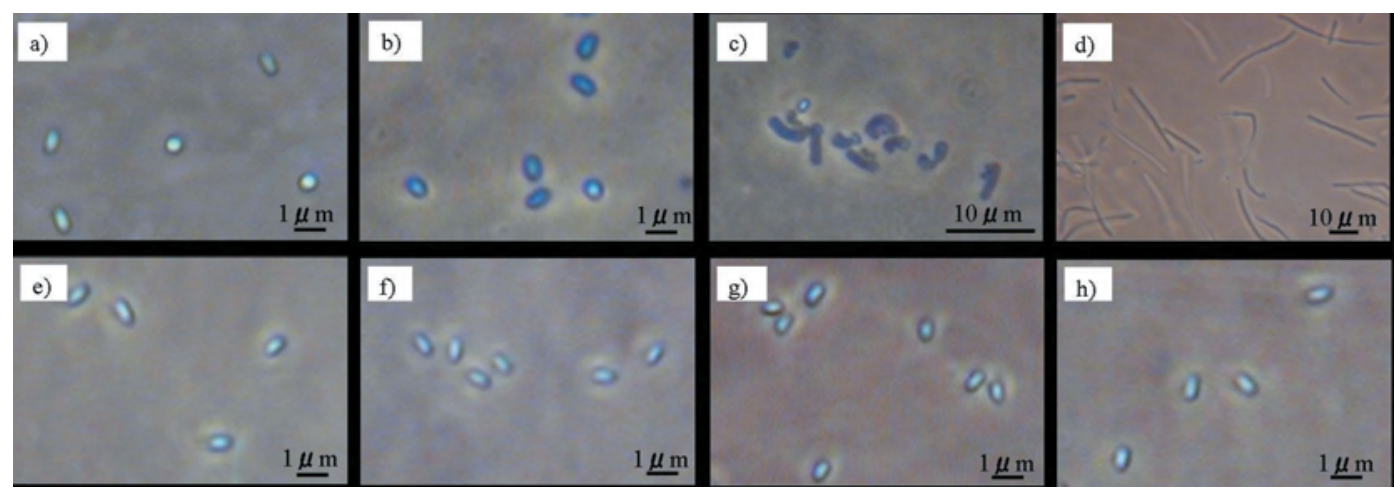

Fig. 5. Phase-contrast microscopic observation of B. subtilis NBRC3007 spores in buffer solution ( $\mathrm{pH} 3.3$ ). (a-d) Non-treated spores: a), control; b), 1 h; c), 2 h; d), 3 h.

(e-h) PEF(12 kV)/HHP(700 MPa) treated spores: e), control; f), 2 h; g), 5 h; h), 24 h. 
the enzymes in the spores responsible for the phase change could be inactivated and germination of the spores could not occur.

\section{Conclusions}

In this study, the effect of HHP or PEF treatment on the inactivation of Bacillus subtilis spores was investigated using suspensions prepared by suspending the spores in a variety of solutions. In addition, the inactivating effect on the spores was investigated by employing HHP and PEF treatments in combination. Furthermore, the viable spore counts were determined and the state of the spores was observed using a phase-contrast microscope, while the spore suspensions were maintained at low temperature after the treatment.

1. The PEF treatment did not substantially reduce the viable spore counts. When the HHP treatment was conducted alone, the lower the $\mathrm{pH}$ of the solution, the more readily the spores were inactivated. The suspension prepared by suspending the spores in $0.5 \mathrm{M}$ acetate buffer solution ( $\mathrm{pH}$ 3.3) exhibited the maximum reduction in viable spore count, i.e., a $6.4 \log$ reduction.

2. When the PEF and HHP treatments were successively performed in that order (PEF/HHP treatment), a significant combined effect on the inactivation of the spores was observed compared with when either the HHP or PEF treatment was conducted alone. The spore suspension in $0.5 \mathrm{M}$ acetate buffer solution ( $\mathrm{pH} 3.3$ ) exhibited the maximum reduction in viable spore count, i.e., a $7.1 \mathrm{log}$ reduction. However, when the spore suspensions using acidic buffer solutions $(\mathrm{pH} \leqq 5.0)$ were successively subjected to HHP and PEF treatments in that order (HHP/PEF treatment), the number of germinated spores was higher than the cases that the HHP treatment was conducted alone.

3. Low-temperature storage of the suspensions after the treatment reduced the occurrence of germination and promoted the inactivation of the spores. In particular, when the spore suspensions in $0.5 \mathrm{M}$ acetate buffer solution ( $\mathrm{pH} 3.3)$ and the orange juice $(\mathrm{pH} 3.9)$ were stored at low temperature after the PEF/HHP treatment, the viable spore counts decreased to non-detectable levels, indicating the complete inactivation of the spores.
4. It was confirmed by phase-contrast microscopy that the non-treated spores gradually turned into phase-dark spores and finally germinated and changed into vegetative cells; on the other hand, the spores subjected to PEF/HHP treatment did not transfer to the phase-dark stage, meaning no germination.

In light of the above, PEF/HHP treatment can be regarded as a revolutionary method of sterilization. More specifically, PEF treatment first damages the spore cells and thereafter, the HHP treatment allows the liquid to penetrate the inside of the spore cells, thereby inactivating the whole spore. For further investigation, the real challenge is to damage the spore cells perfectly by PEF treatment rather than to achieve perfect pressure inactivation of spores by HHP treatment. In addition, it is necessary to find a pressure level required to irreversibly denature the enzymes responsible for the germination. It is expected that the full potential of this perfect nonthermal sterilization will be realized when the abovementioned problems are solved.

\section{Acknowledgements}

This work was a part of the findings obtained from our study on the "Development of sterilization technique for food products using a combination of high-voltage pulsed electric field and hydrostatic high-pressure treat ments," which was supported by the General Food Policy Bureau of the Ministry of Agriculture, Forestry and Fisheries of Japan through their project of technical development for increasing the supply of food products, "Brand Nippon", in 2004. The authors sincerely thank the members of the Research Institute of Echigoseika Co., Ltd. for supporting this work.

\section{References}

[1] A. Yamazaki, A. Sasagawa, M. Kinefuchi, A. Yamada: in High pressure Bioscience, eds. S. Kunugi, S. Shimada, A. Suzuki, R. Hayashi (San-Ei Pub., Kyoto, 1994), Chap. 41, pp. 328-335, in Japanese.

[2] E.Y. Wuytack, C.W. Michiels: Int. J. Food Microbiol., 64, 333 (2001).

[3] Y. Taki, T. Awao, N. Mitsuura, Y. Takagaki: in Pressure-processed food, ed. R. Hayashi (San-Ei Pub., 
Kyoto, 1990), Chap. 12, pp. 143-155, in Japanese.

[4] Y. Yonemoto, T. Yamashita, M. Muraji, W. Tatebe, H. Ooshima, J. Kato, A. Kimura, K. Murata: J. Ferment Bioeng., 75, 99 (1993).

[5] V.O. Marquez, G.S. Mittal, M. W. Griffiths: J. Food Sci., 62, 399 (1997).

[6] S. Furukawa, T. Watanabe, T. Tai, J. Hirata, N. Narisawa, T. Kawarai, H. Ogihara, M. Yamasaki: Int. J. Food Microbiol., 91, 209 (2004).

[7] H.C. Deeth, N. Datta: Food Aust., 54, 273 (2002).

[8] S. Spilimbergo, A. Bertucco, F. Dehgani, N.R. Foster: Biotechnol. Bioeng., 82, 118 (2003).

[9] A. Nakayama, Y. Yano, S. Kobayashi, M. Ishikawa,

K. Sakai: Appl. Environ. Microbiol., 62, 3897 (1996).

[10] H. Kusaoke, Y. Hayashi, Y. Kadowaki, H. Kimoto: Agric. Biol. Chem., 53, 2441 (1989).

[11] T. Ohshima, M. Sato: Journal of Cookery Science of Japan, 36, 334 (2003), in Japanese.

[12] T. Ohshima, K. Okuyama, M. Sato: Journal of Electrostatics, 55, 227 (2002).

[13] N.M. Ishida, T. Ohshima, M. Sato: Japan J. Food Eng., 5, 35 (2004), in Japanese.

[14] L. Marsili, S Espie, J.G. Anderson, S.J. Macgregor: Radiat. Rhys. Chem., 65, 507 (2002)

[15] R.G.K. Leuschner, P.J. Lillford: Lett. Appl. Microbiol., 29, 228 (1999).

[16] N.M. Ishida, A.T. Sugiarto, T. Ohshima, M. Sato: Japan J. Food Eng., 4, 47 (2003), in Japanese.
[17] V. Heinz, S.T. Phillips, M. Zenker, D. Knorr: Food Biotechnol., 13, 155 (1999).

[18] S.Y. Ho, G.S. Mittal, M.W. Griffiths, J.D. Cross: J. Food Sci., 60, 1337 (1995).

[19] N.R. Reddy, H.M Solomon, G.A. Fingerhut, V.M. Balasubramniam, E.J. Rhodehamel, S. Palaniappan: J. Food Safety, 19, 227 (1999).

[20] T. Grahl, H. Markl: Appl. Microbiol. Biotechnol., 45, 148 (1996).

[21] S. Bendicho, A.R. Marselles-Fontanet, G.V. Barbosa-Canovas, O. Martin-Belloso: J. Food Eng., 69, 317 (2005).

[22] S.Y. Ho, G.S. Mittal, J.D. Cross: J. Food Eng., 31, 69 (1997).

[23] I. Hayakawa, H. Horiuchi, A. Mizunaga, S. Furukawa, T. Nakashihtema, Y. Fujio: Nippon Shokuhin Kagaku Kogaku Kaishi, 45, 158 (1998), in Japanese.

[24] I. Hayakawa, S. Furukawa, A. Mizunaga, H. Horiuchi, T. Nakashima, Y. Fujio, Y. Yano, T. Ishikura, K. Sasaki: J. Food Sci., 63, 371 (1998).

[25] S.E. Wallen, H.W. Walker: J. Food Sci., 45, 605 (1980).

[26] R.G.K. Leuschner, D.P. Ferdinando, P.J. Lillford: Colloids Surf. B., 19, 31 (2000).

[27] A. Atrih, P. Zollner, G. Allmaier, M.P. Williamson, S.J. Foster: J. Bacteriology, 180, 4603 (1998).

[Received October 17, 2005] 